\title{
Radiografía Panorámica: Una Herramienta Invaluable para el Es- tudio del Componente Óseo y Dental del Territorio Maxilofacial
}

\author{
Panoramic Radiographs: An Invaluable Tool for the Study \\ of Bone and Teeth Components in the Maxillofacial Region
}

\author{
Ramón Fuentes $^{1,2}$; Alain Arias ${ }^{1,2}$ \& Evelyn Borie-Echevarría ${ }^{3}$
}

FUENTES, R.; ARIAS, A. \& BORIE-ECHEVARRÍA, E. Radiografía panorámica: una herramienta invaluable para el estudio del componente óseo y dental del territorio maxilofacial. Int. J. Morphol., 39(1):268-273, 2021.

RESUMEN: La radiografía panorámica es probablemente el examen de rutina más solicitado por los dentistas en el mundo. Entre sus ventajas están su bajo costo, rapidez y mayor seguridad debido a que los formatos digitales han permitido disminuir las dosis y tiempos de exposición a la radiación. Su potencial como herramienta diagnóstica radica en la posibilidad que tiene el clínico de observar el territorio maxilofacial casi en su totalidad, pudiendo realizar un estudio anatómico y morfológico acabado de diferentes regiones. En esta revisión se quiere ofrecer una mirada general de la amplia gama de usos que se le pueden dar a la radiografía panorámica, tanto en la clínica como en el ámbito de la investigación, destacando su potencial como herramienta de diagnóstico y su importancia para algunas áreas de especialidad en odontología.

PALABRAS CLAVE: Radiografía panorámica; Maxilar; Mandíbula; Patologías óseas; Calcificaciones.

\section{INTRODUCCIÓN}

La ortopantomografía o radiografía panorámica es una de las herramientas diagnósticas más utilizadas por los odontológos en la práctica clínica regular. La técnica permite una visualización conjunta de la maxila y la mandíbula en una sola placa basándose en la combinación de la radiografía con haz de hendidura y los principios de la tomografía (Hirschmann, 1987). Entre sus ventajas está el bajo costo, lo cual permite su uso masivo como herramienta diagnóstica y epidemiológica, su relativo bajo nivel de radiación y su considerable poder de resolución, el cual depende de la correcta posición del paciente y de las estructuras que se desean visualizar (Rushton \& Horner, 1996). Desde la introducción de las técnicas digitales en la radiografía intra- y extra-oral, se han logrado importantes avances en la mejoría de definición y resolución de la imagen, incluso reduciendo el tiempo de exposición a los rayos $\mathrm{X}$ y con el ello el riesgo a un daño acumulativo (McDavid et al., 1995).

Debido a su capacidad para proyectar completamente las estructuras óseas maxilofaciales, tanto la radiografía panorámica convencional como la técnica digital se han utilizado con fines clínicos en el diagnóstico y también para la investigación. En este último aspecto, el uso de la radiografía panorámica ha permitido una amplia caracterización de estructuras anatómicas e incluso de procesos fisiopatológicos, facilitando que el clínico posea un mayor y mejor conocimiento del territorio estomatognático. En esta revisión se exponen los principales usos que se han dado a la radiografía panorámica en el ejercicio de la odontología e investigación, destacando su potencial como herramienta diagnóstica y de estudio, y sobre todo para algunas áreas de especialidad en odontología.

Estudio de los arcos dentarios. Desde su aparición, la radiografía panorámica ha permitido el estudio simultáneo de los arcos dentarios, tanto maxilar como mandibular, en forma rápida y sencilla como ninguna otra técnica lo había permitido antes. Con ello, la identificación de alteraciones en la morfología dental se hizo cada vez más evidente para el clínico y por tanto más prevalentes en la población.

A través de radiografías panorámicas digitales ha sido posible la identificación de variaciones anatómicas de la raíz dental, incluyendo raíces dobles y triples en premolares

\footnotetext{
${ }^{1}$ Departamento de Odontología Integral Adultos, Facultad de Odontología, Universidad de La Frontera, Temuco, Chile.

${ }^{2}$ Centro de Investigación en Ciencias Odontológicas, Facultad de Odontología, Universidad de La Frontera, Temuco, Chile.

${ }^{3}$ Escuela de Odontología, Facultad de Ciencias, Universidad Mayor, Chile.
} 
(Oporto et al., 2010, 2013) y raíces dobles en caninos (Fuentes $\&$ Borie, 2013). Asimismo, en cuanto a las raíces dentarias, con la radiografía panorámica se han podido realizar estudios detallados sobre las dilaceraciones, las cuales ocurren con relativa frecuencia y que pueden significar complicaciones para la endodoncia, cirugía e implicar esfuerzos adicionales para el movimiento ortodóncico. De este modo, en un estudio con 294 premolares mandibulares analizados a través de radiografía panorámica se reportó que más del $70 \%$ de estos dientes posee algún grado de curvatura en sus raíces, que estás se originan principalmente en el tercio apical $(72,25 \%)$ y que poco más de la mitad $(58,84 \%)$ presenta una curvatura con orientación distal (Fuentes et al., 2015). Otro estudio en el que se analizaron las raíces de 412 dientes, se encontró que el $84,72 \%$ presentaba algún tipo de curvatura mientras que el $0,73 \%$ mostraba una clara dilaceración con un ángulo mayor de 90 grados (Fuentes et al., 2018a). Otra alteración de las raíces dentarias susceptible de ser pesquisada a través de la radiografía panorámica es el enanismo radicular, pues permite la comparación con dientes vecinos y de arcadas contrarias estableciendo parámetros inmediatos para la valoración del tamaño de la raíz. Su diagnóstico resulta importante para la realización de movimientos ortodóncicos, sobre todo en aquellos casos severos en que hay muchos dientes afectados con esta alteración, pues se ha visto una tendencia a incrementar el riesgo de reabsorción radicular (Valladares Neto et al., 2013).

Otra de las típicas alteraciones dentarias que se pueden identificar y que son posibles de estudiar utilizando la radiografía panorámica son las anomalías de número. Los dientes supernumerarios o hiperodoncia es la presencia de uno o más dientes adicionales a la formula normal de $20 \mathrm{o}$ 32 de la dentición primaria o permanente, respectivamente (Valmaseda-Castellón et al., 2001). La prevalencia de dientes supernumerarios es relativamente baja y varía de acuerdo a las características de la población estudiada, pudiendo presentarse tanto en niños con dentición mixta o incluso en adultos desdentados, en donde su identificación por lo general es accidental (Fuentes et al., 2018b). Otra anomalía de número en la formula dental es la agenesia, ausencia congénita o hipodoncia. La agenesia dental afecta principalmente a la dentición permanente y su prevalencia es mayor que la de hiperodoncia (Lozada, 2003). En un estudio sistemático de los registros dentales pertenecientes a 307 niños de entre 6 a 11 años atendidos en una clínica odontológica universitaria, y que incluyó el análisis de radiografías panorámicas convencionales, se reportó que la prevalencia de individuos con agenesia fue de un 4,2\%, siendo los segundos premolares los más afectados (Pineda et al., 2011).Estudios como estos son de gran utilidad para la odontopediatría pues permiten mantener una actitud de vigilancia en niños que van a ser sometidos a tratamientos de guía de erupción u ortodoncia interceptiva. En casos raros o poco frecuentes como pacientes no-sindrómicos y con múltiples agenesias, la radiografía panorámica ofrece la oportunidad de visualizar todo el sistema maxilofacial y de esta forma evaluar eventuales consecuencias en el resto de estructuras, sobre todo cóndilos y articulación témporomandibular (ATM) (Borie et al., 2012).

Por último, a través de la radiografía panorámica también es posible identificar y dar seguimiento al desarrollo normal o patológico de los dientes en diferentes zonas de la maxila y mandíbula. Casos poco habituales como la erupción de cuartos molares (Fuentes et al., 2012) o la erupción ectópica de terceros molares (Fuentes \& Oporto, 2009) pueden ser pesquisados tempranamente en evaluaciones radiográficas rutinarias y ser de gran utilidad en la planificación de tratamientos quirúrgicos posteriores.

Estudio de la Maxila y el Seno Maxilar. Mediante la examinación rutinaria de la radiografía panorámica es posible identificar diversos tipos de anomalías que afectan a los huesos maxilar y mandibular. En el primer caso, las anomalías factibles de pesquisar se limitan generalmente a la presencia de lesiones de diversa índole en los senos paranasales y, muy principalmente, en el seno maxilar. La radiografía panorámica permite la identificación, sobre todo, de cuerpos radioopacos dentro de las amplias zonas radiolúcidas de los senos y que, según su ubicación, extensión y forma, podrían requerir de otros exámenes, como la tomografía computarizada cone beam, para el diagnóstico diferencial. Por ejemplo, en la literatura abundan los reportes de casos sobre hallazgos poco habituales en el interior del seno maxilar, tales como exostosis (Borie et al., 2014), quistes (Fuentes et al., 2008) e incluso cuerpos extraños de origen exógeno (Arellano-Villalón et al., 2020). Más recientemente, mediante la evaluación de la visibilidad de la línea innominada en la radiografía panorámica ha sido posible estudiar la presencia de depresiones de la pared anterior del seno maxilar, lo cual se asocia a algunas patologías tales como el síndrome del seno silente, una condición poco descrita en la literatura, generalmente asintomática pero asociada con trastornos en el rostro, mandíbula, dedos y pezones (Yoshidaet al., 2017; Serindere \& Aktuna Belgin, 2020).

Estudio de la Mandíbula: forámenes, canales y patologías del desarrollo. La mandíbula es probablemente la estructura ósea más estudiada a través de la radiografía panorámica. Con ella ha sido posible estudiar sus cambios morfológicos que ocurren durante el envejecimiento y así establecer parámetros útiles para la planificación de tratamientos prostodóncicos. Por ejemplo, se ha visto que en cuanto al ángulo mandibular no existen diferencias entre sujetos dentados y no dentados (Ceylan et al., 1998), sin embargo, otros parámetros como la altura de la rama, altura condilar o gro- 


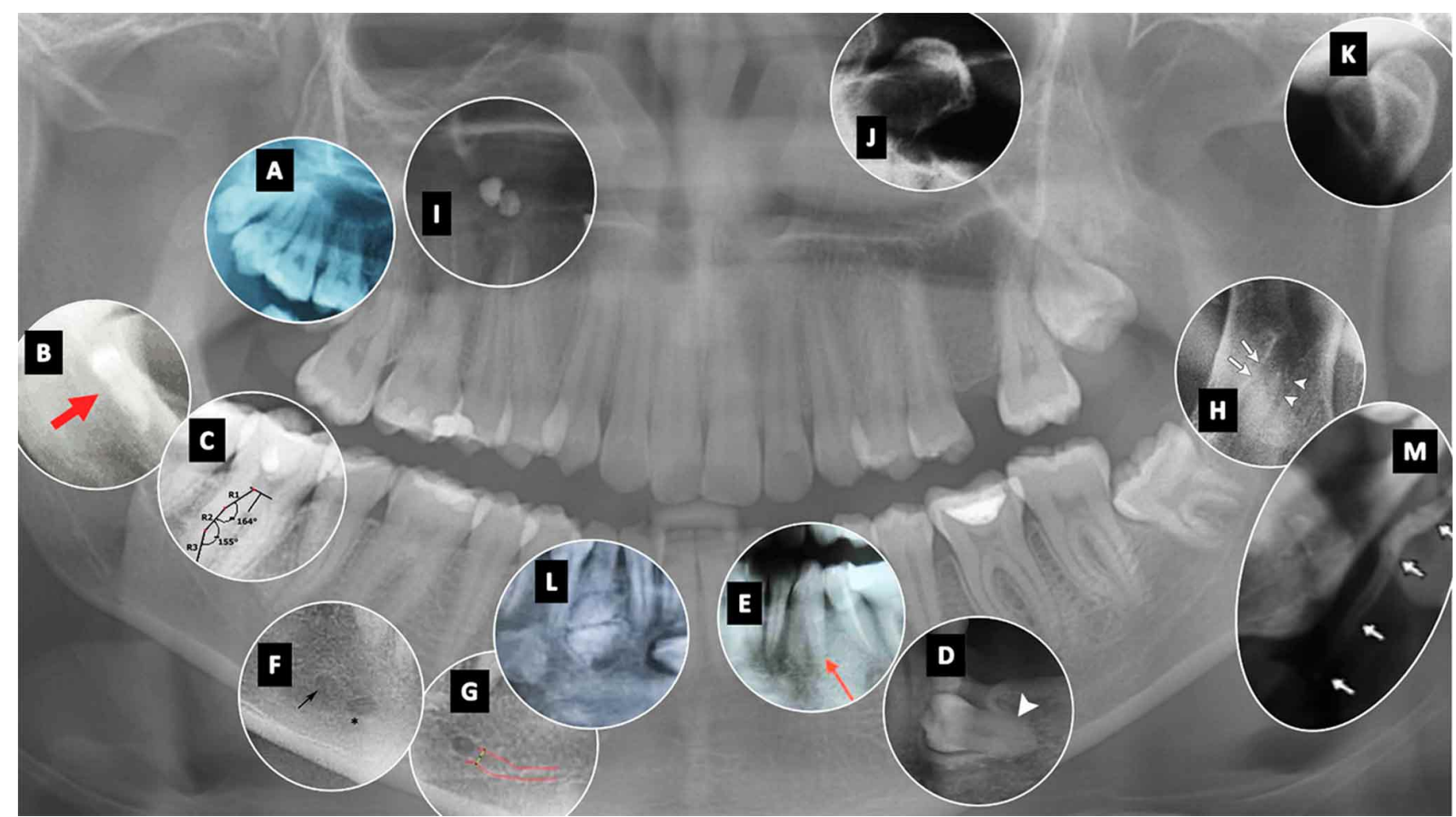

Fig. 1. La radiografía panorámica, tanto convencional como digital, es una excelente herramienta para la pesquisa y diagnóstico de diferentes condiciones que pueden afectar a los arcos dentarios y a los dientes (A-E), el cuerpo mandibular incluyendo forámenes y canales (F-H), el hueso maxilar y especialmente al seno maxilar (I, J), los cóndilos de la ATM (K), algunas patologías tales como odontomas y otros tumores $(\mathrm{L})$ e incluso calcificaciones raras de ligamentos y estructuras vasculares $(\mathrm{M})$.

sor del hueso cortical, sí presentan diferencias de acuerdo al estado dentario siendo más marcado en mujeres que en hombres (Joo et al., 2013). Incluso, la radiografía panorámica ha sido utilizada para caracterizar la morfología mandibular de sujetos con Síndrome de Down (Satir, 2019) o sujetos bajo tratamiento con quimioterapéuticos (Busra \& Guldane, 2019).

Dentro de la mandíbula, el canal mandibular es una estructura a tener muy en cuenta sobre todo en procedimientos quirúrgicos y para la colocación de implantes dentales. Su apropiada localización y caracterización de su recorrido permitirá al clínico planificar adecuadamente las intervenciones y así evitar daños al paquete vasculo-nervioso. En la literatura abundan los reportes de caso de canales mandibulares dobles o bífidos, el cual, según la extensión y ubicación delcanal adicional, requerirá de exámenes de mayor resolución (Fuentes et al., 2018c). En un estudio realizado en 925 radiografías panorámicas digitales se reporto que la prevalencia de canal mandibular bífido fue de un 11 $\%$, siendo más común en sujetos jóvenes y sin diferencias según sexo (Fuentes et al., 2018d). En este mismo estudio también se evaluó la presencia del canal retromolar, un canal accesorio que transcurre superior y lateral al canal mandibular, encontrándose una prevalencia de tan sólo un $0,9 \%$. Otro tipo de variación factible de ser pesquisaba mediante la radiografía panorámica es el denominado canal de Serres, descrito por primera vez en 1817 y que corresponde a un canal altamente desarrollado en el feto que transcurre inferior al canal mandibular y que posee una arteria independiente (Serres, 1817). En un estudio que analizó 495 radiografía panorámicas, se encontró una prevalencia de este canal del 10,51\%, siendo más predominante en sujetos menores de 20 años (Fuentes et al., 2016a). Otro elemento importante en la caracterización del canal mandibular, es la ubicación de su foramen de entrada o foramen mandibular. En un análisis 927 radiografías, se determinó que el área, altura y ancho promedio del foramen mandibular es de 6,37 $\mathrm{mm}^{2}, 2,27 \mathrm{~mm}$ y $3,19 \mathrm{~mm}$ respectivamente, mientras que la prevalencia del foramen mandibular doble fue de 1,51\% (Farfán et al., 2020).

Otro de los reparos anatómicos de la mandíbula frecuentemente visualizados a través de la radiografía panorámica es el foramen mental, pues según algunos estudios su capacidad para detectarlo supera el $95 \%$ (al Jasser \& Nwoku, 1998; Ngeow \& Yuzawati, 2003). El foramen mental se encuentra en la pared bucal del cuerpo de la mandíbula y re- 
presenta el término del canal mental y mandibular. De acuerdo a un estudio realizado en 215 radiografías panorámicas convencionales, el foramen mental fue posible de identificar en casi el $88 \%$ de los casos, describiendo una posición en sentido anteroposterior mas cercana al segundo premolar en el lado derecho y un entre ambos premolares en el lado izquierdo (Fuentes et al., 2014). Otro estudio similar analizó 163 radiografías panorámicas digitales y encontró igualmente que la posición más común en ambos lados de la mandíbula es entre ambos premolares y bajo el nivel de los ápices (Fuentes et al., 2017a). Adicionalmente, establecieron algunas medidas morfométricas que relacionaban la posición del foramen mental con algunos puntos de referencia, encontrando que en promedio el foramen se encuentra aproximadamente a $25 \mathrm{~mm}$ de la línea media mandibular y a $12 \mathrm{~mm}$ del margen inferior de la mandíbula, para ambos lados (Fuentes et al., 2017a). Asimismo, con la radiografía panorámica se ha podido determinar que el área, altura y ancho promedio del foramen mental es de $5,46 \mathrm{~mm}^{2}, 2,77$ $\mathrm{mm}$ y $2,57 \mathrm{~mm}$ respectivamente, mientras que la prevalencia de foramen mental doble fue de $2,58 \%$ (Farfán et al.).

El canal incisivo mandibular es una extensión hacia anterior del canal mandibular desde el foramen mental que contiene un paquete vasculo-nervioso que inerva e irriga a los dientes anteriores (Romanos et al., 2012). Su identificación es importante a la hora de realizar procedimientos de anestesia, cirugía periapical o en implantología. Varios son los reportes que dan cuenta de la importancia de su localización antes de realizar procedimientos quirúrgicos en la zona (Beltrán et al., 2011). Se ha reportado que su prevalencia podría alcanzar poco más del $50 \%$, siendo más predominante en hombres y cercano a los primeros premolares y caninos (Fuentes et al., 2017b).

Estudio de los Cóndilos Mandibulares y la ATM. Más allá del estudio de los huesos maxilares y la mandíbula, con la radiografía panorámica también es posible estudiar los cóndilos de la ATM. Al respecto, una de las evaluaciones más recurrentes es la valoración de la simetría o asimetría condilar utilizando medidas que comparan las alturas del cóndilo en ambos lados. Muy empleado es el método descrito por Kjellberg et al. (1994). Utilizando este método, un estudio que evaluó 235 radiografías panorámicas digitales determinó que un alto porcentaje presenta simetría (Fuentes et al., 2018e), mientras que otro estudio que analizó 48 radiografías panorámicas convencionales de pacientes con signos y síntomas de trastorno temporomandibular reportó que el 54,2\% de los casos presentaban asimetría (Iturriaga et al., 2012). Por otro lado, la radiografía panorámica también ha sido útil para el diagnóstico de patologías poco frecuentes que afectan a los cóndilos y que pueden provocar trastornos más severos si no son tratados a tiempo. Tal es el caso del cóndilo bífido, por ejemplo, el cual puede adoptar formas tan dispares como la presencia de un cóndilo bigeminado en el plano latero-medial o la presencia de dos procesos condilares, uno anterior y otro posterior, en sentido sagital (Fuentes et al., 2009). En estos casos, la radiografía panorámica podría representar una herramienta de cribaje para disfuncionistas y que con el análisis de los cóndilos se podrían establecer parámetros de predicción de trastornos en la ATM.

Estudio de otras Patologías. La radiografía panorámica no solo permite el estudio normal de la morfología ósea si no que también ofrece una oportunidad para el diagnóstico de patologías de diversa índole y que se manifiestan afectando la radiodensidad del hueso. Ejemplos clásicos son el hallazgo radiográfico casual de osteoesclerosis en el hueso mandibular. Un amplio estudio realizado en 957 radiografías panorámicas digitales permitió la identificación de 29 casos en 27 individuos, la mayoría ubicándose más allá del nivel apical de los dientes (Fuentes et al., 2018f).

También, mediante la examinación rutinaria de las radiografías panorámicas es posible encontrar indicios de otro tipo de patologías tales como la presencia de lesiones periapicales, incluyendo quistes y granulomas, los cuales según su apariencia requerirán de radiografías periapicales para valorar mejor su relación con dientes vecinos y determinar el tratamiento endodóntico y periodontal más adecuado (Fuentes et al., 2019).

Otro hallazgo de relativa frecuencia es la presencia de tumores benignos tales como el odontoma (Salamanca et al., 2017) o el mixomaodontogénico (Dotta et al., 2020), los cuales, si no son se detectan a tiempo, al crecer podrían causar desplazamientos de dientes o compresión de paquetes neurovasculares. Incluso, la radiografía panorámica debe pensarse como una herramienta para la pesquisa de lesiones tumorales benignas y cáncer. En todos estos casos la radiografía panorámica se perfila como una herramienta para el control periódico de patologías.

Calcificaciones Ligamentosas y Vasculares. Finalmente, es preciso mencionar algunas de las aplicaciones de la radiografía panorámica menos considerada por los dentistas en la práctica clínica y que pueden tener repercusiones a nivel sistémico del paciente. Hablamos de las calcificaciones que pueden ocurrir en ligamentos y en regiones vasculares. Por ejemplo, se ha reportado que el proceso estiloides podría verse elongado en la radiografía panorámica dando lugar a la sospecha de que el ligamento estilohioideo podría estar parcialmente calcificado (Fuentes et al., 2016b). Es más, en un estudio en el que se analizaron 968 radiografías panorámicas de sujetos de ambos sexos 
se encontró que el 3,61 \% presentaba procesos estiloides elongados, siendo mujeres la mayoría de los casos y con una longitud promedio que varía entre los 42 y $50 \mathrm{~mm}$ (Fuentes et al., 2007).

Por otro lado, la radiografía panorámica ha demostrado ser una excelente herramienta para el diagnóstico de calcificaciones en la arteria carotidea. La relevancia en la identificación de estos hallazgos en la arteria carótida radica en que podría tratarse de placas de ateroma calcificadas las cuales pueden ser un factor predictivo para el desarrollo de accidentes cerebrovasculares (Mupparapu \& Kim, 2007). En un meta-análisis reciente, se concluyó que la radiografía panorámica posee una excelente sensibilidad y una buena especificidad con una precisión diagnóstica que fue buena o excelente en el $50 \%$ de los casos (Schroder et al., 2019). Con este estudio es posible también concluir que la radiografía panorámica podría utilizarse como una herramienta de cribaje secundaria para este tipo de pacientes permitiendo realizar actividades de prevención en la población.

\section{CONCLUSIONES}

El potencial de la radiografía panorámica ya sea en su formato convencional o digital es amplio y no solo se limita al diagnóstico de patología que competen al odontólogo, sino que también a otros profesionales de la medicina. Su potencial radica en su capacidad de ofrecer una vista global o general de las estructuras maxilofaciales, que a pesar de sus limitaciones en cuanto en magnificación y distorsión, la convierten en una herramienta diagnóstica de gran versatilidad.

FUENTES, R.; ARIAS, A. \& BORIE-ECHEVARRÍA, E. Panoramic radiographs: An invaluable tool for the study of bone and teeth components in the maxillofacial region. Int. J. Morphol., 39(1):268-273, 2021

SUMMARY: The panoramic radiograph is in all likelihood, the routine exam most required by dentists in the clinic worldwide. Among the advantages of using this type of radiograph, is that it is inexpensive, quick and also safer in digital format since it reduces the radiation dose and time of exposure. Its potential as a diagnostic tool is based on the possibility to explore almost all of the maxillofacial area, allowing a proper anatomic and morphological study of different areas at the same time. In this review, we aimed to provide an overview of the multiple uses of panoramic radiographs, highlighting its potential as a diagnostic tool and also its importance in some specialized areas of dentistry.

KEY WORDS: Panoramic radiographs; Maxilla; Mandible;Bone pathologies; Calcifications.

\section{REFERENCIAS BIBLIOGRÁFICAS}

al Jasser, N. M. \& Nwoku, A. L. Radiographic study of the mental foramen in a selected Saudi population. Dentomaxillofac. Radiol., 27(6):341-3, 1998.

Arellano-Villalón, M.; Navarro, P.; Arias, A. \& Fuentes, R. Radiographic finding of a foreign body in the maxillary sinus floor of an asymptomatic subject. Int. J. Odontostomat., 14(2):150-3, 2020.

Beltrán, V.; Cantín, M.; Fuentes, F. R. \& Engelke, W. Bilateral presence of mandibular incisive canal. an anatomical structure with clinical relevance. Int. J. Morphol., 29(2):543-9, 2011.

Borie, E.; Fuentes, R. \& Beltrán, V. Multiple tooth agenesis in nonsyndromic patient: a rare case report. Int. J. Morphol., 30(2):634-6, 2012.

Borie, E.; Watanabe, P.; Orsi, I. \& Fuentes, R. Idiopathic bilateral antral exostoses: a rare case in maxillary sinus. Int. J. Surg. Case Rep., 5(9):624-7, 2014.

Busra, T. \& Guldane, M. Evaluation of mandibular morphometry in the bisphosphonate users. Int. J. Morphol., 37(2):654-63, 2019.

Ceylan, G.; Yaníkoglu, N.; Yílmaz, A. B. \& Ceylan, Y. Changes in the mandibular angle in the dentulous and edentulous states. J. Prosthet. Dent., 80(6):680-4, 1998.

Dotta, J. H.; Miotto, L. N.; Spin-Neto, R. \& Ferrisse, T. M. Odontogenic myxoma: systematic review and bias analysis. Eur. J. Clin. Invest., 50(4):e13214, 2020.

Farfán, C.; Arias, A.; Dias, F.; Garay, I.; Navarro, P. \& Fuentes, R. Prevalence and morphometric analysis of double mental foramen and double mandibular foramen from digital panoramic radiographs. Int. J. Morphol., 38(3):714-9, 2020.

Fuentes, F. R. \& Oporto, V. G. Ectopic and Impacted Third Molar in Retromolar Zone. A Case Report. Int. J. Morphol., 27(1):35-8, 2009.

Fuentes, F. R.; Garay, C. I. \& Borie, E. E. Presence of mucus retention cysts in maxillary sinus detected by panoramic radiographs on patients of Temuco, Chile. Int. J. Morphol., 26(3):697-9, 2008.

Fuentes, F. R.; Oporto, V. G.; Garay, C. I.; Bustos, M. L.; Silva, M. H. \& Flores, F. H. Styloid process in the panoramics radiographic sample of Temuco-Chile City. Int. J. Morphol., 25(4):729-33, 2007.

Fuentes, R. \& Borie, E. Bilateral two-rooted mandibular canines in the same individual: a case report. Int. J. Odontostomat., 7(3):471-3, 2013

Fuentes, R.; Álvarez, G.; Arias, A.; Borie-Echeverría, E. \& Dias, F. Apical periodontitis: histological and morphometric characterization of radicular cysts and periapical granulomas. Int. J. Morphol., 36(4):1268$74,2018 \mathrm{~d}$.

Fuentes, R.; Álvarez, G.; Garay, I.; Arias, A. \& Dias, F. J. Supplementary supernumerary teeth in a partially edentulous adult patient: case report. Int. J. Morphol., 36(2):478-82, 2018b.

Fuentes, R.; Arellano-Villalón, M.; Soto-Faúndez, N.; Dias, F. J.; Navarro, P. \& Arias, A. Condylar and mandibular symmetry indexes through digital panoramic radiographs in a sample of Chileans patients. Int. J. Morphol., 36(3):854-8, 2018e.

Fuentes, R.; Arias, A.; Astete, N.; Farfán, N.; Garay, I. \& Dias, F. Prevalence and morphometric analysis of idiopathic osteosclerosis in a Chilean population. Folia Morphol. (Warsz.), 77(2):272-8, $2018 \mathrm{f}$.

Fuentes, R.; Arias, A.; Bucchi, C.; Saravia, D. \& Dias, F. Prevalence and morphometric characteristics of the mandibular incisive canal through panoramic radiographs in a Chilean population. Int. J. Morphol. 35(3):931-7, 2017b

Fuentes, R.; Arias, A.; Farfán, C.; Astete, N.; Garay, I.; Navarro, P. \& Dias, F. Morphological variations of the mandibular canal in digital panoramic radiographs: a retrospective study in a Chilean population. Folia Morphol. (Warsz.), 78(1):163-70, 2019.

Fuentes, R.; Arias, A.; Navarro, P. \& Bucchi, C. Morphological study of the mandible using digital panoramic X-rays, Part I: The canal of Serres, prevalence and location. J. Anat. Soc. India, 65(2):69-74, 2016 a. 
Fuentes, R.; Arias, A.; Navarro, P.; Ottone, N. \& Bucchi, C. Morphometric study of mandibular premolars in panoramic digital radiographs; analysis of root curvatures. Int. J. Morphol., 33(2):476-82, 2015.

Fuentes, R.; Borie, E. \& Beltrán, V. Radiographical and macroscopical visualization of fourth molars: a report of four cases in maxilla. Int. J. Morphol., 30(1):115-8, 2012.

Fuentes, R.; Cantín, M.; Navarro, P.; Borie, E.; Beltrán, V. \& Bucchi, C. Characterization of anatomical structures using panoramic radiographs: the mental foramen. Int. J. Morphol., 32(4):1423-9, 2014.

Fuentes, R.; Farfán, C.; Astete, N.; Garay, I.; Dias, F. \& Arias, A. Bilateral bifid mandibular canal: a case report using cone beam computed tomography. Folia Morpho.l (Warsz.), 77(4):780-4, 2018c.

Fuentes, R.; Farfán, C.; Astete, N.; Navarro, P. \& Arias, A. Distal root curvatures in mandibular molars: analysis using digital panoramic $\mathrm{X}$ rays. Folia Morphol. (Warsz)., 77(1):131-7, 2018a.

Fuentes, R.; Flores, H.; Silva, H. \& Flores, T. Bifid condylar process. Cases report. Int. J. Morphol., 27(2):539-41, 2009.

Fuentes, R.; Flores, T.; Dias, F.; Farfán, C.; Astete, N.; Navarro, P. \& Arias, A. Localization of the mental foramen through digital panoramic radiographs in a Chilean population. Int. J. Morphol., 35(4):1309-15, 2017a.

Fuentes, R.; Saravia, D.; Garay, I. \& Ottone, N. Asymptomatic bilateral calcified stylohyoid ligaments detection by panoramic radiography and cone beam computerized tomography. Biomed. Res. (India)., 27(4):1-3, $2016 \mathrm{~b}$.

Hirschmann, P. N. The current status of panoramic radiography. Int. Dent. J., 37(1):31-7, 1987.

Iturriaga, V.; Navarro, P.; Cantín, M. \& Fuentes, R. Prevalence of vertical condilar asymmetry of the temporomandibular joint in patients with signs and symptoms of temporomandibular disorders. Int. J. Morphol., 30(1):315-21, 2012.

Joo, J. K.; Lim, Y. J.; Kwon, H. B. \& Ahn, S. J. Panoramic radiographic evaluation of the mandibular morphological changes in elderly dentate and edentulous subjects. Acta Odontol. Scand., 71(2):357-62, 2013.

Kjellberg, H.; Ekestubbe, A.; Kiliaridis, S. \& Thilander, B. Condylar height on panoramic radiographs. A methodologic study with a clinical application. Acta Odontol. Scand., 52(1):43-50, 1994.

Lozada, O. Alteraciones en el Número de Dientes Asociados a Maloclusiones. Tesis de Postgrado de Ortodoncia. Caracas, Facultad de Odontología, Universidad Central de Venezuela, 2003.

McDavid, W.; Welander, U.; Brent Dove, S. \& Tronje, G. Digital imaging in rotational panoramic radiography. Dentomaxillofac. Radiol., 24(2):68$75,1995$.

Mupparapu, M. \& Kim, I. H. Calcified carotid artery atheroma and stroke: a systematic review. J. Am. Dent. Assoc., 138(4):483-92, 2007.

Ngeow, W. C. \& Yuzawati, Y. The location of the mental foramen in a selected Malay population. J. Oral Sci., 45(3):171-5, 2003.

Oporto, G.; Saavedra, R.; Soto, C. \& Fuentes R. Double root anatomical variations in a single patient: endodontic treatment and rehabilitation of a three-rooted first premolar. Case report. Int. J. Morphol., 31(1):45-9, 2013.

Oporto, V. G. H.; Fuentes, R. E. \& Soto, P. C. C. Tooth root and pulp canal anatomical variations. Int. J. Morphol., 28(3):945-50, 2010.

Pineda, P.; Fuentes, R. \& Sanhueza, A. Prevalence of dental agenesis in children with mixed dentition of teaching assistant dental clinics at the Universidad de La Frontera. Int. J. Morphol., 29(4):1087-92, 2011.

Romanos, G. E.; Papadimitriou, D. E.; Royer, K.; Stefanova-Stephens, N.; Salwan, R.; Malmström, H. \& Caton, J. G. The presence of the mandibular incisive canal: a panoramic radiographic examination. Implant. Dent., 21(3):202-6, 2012.

Rushton, V. \& Horner, K. The use of panoramic radiology in dental practice. J. Dent., 24(3):185-201, 1996.

Salamanca, C.; Ottone, N.; Arias, R.; Garay, I. \& Fuentes, R. Transmigrated canines associated to odontomas. Int. J. Morphol., 35(2):465-8, 2017.

Satir, S. Determination of mandibular morphology in a TURKISH population with Down syndrome using panoramic radiography. BMC Oral Health., 19(1):36, 2019.
Schroder, A. G. D.; de Araujo, C. M.; Guariza-Filho, O.; Flores-Mir, C.; de Luca Canto, G. \& Porporatti, A. L. Diagnostic accuracy of panoramic radiography in the detection of calcified carotid artery atheroma: a metaanalysis. Clin. Oral Investig., 23(5):2021-40, 2019.

Serindere, G. \& Aktuna Belgin, C. An evaluation of the relationship between maxillary sinus anterior wall depression and maxillary sinus opacity by computed tomography and panoramic radiography. Oral Radiol., 36(4):383-8, 2020.

Serres, A. E. R. A. Essai Sur l'Anatomie et la Physiologie des Dents, Ounovellethéorie de la Dentition. Paris, Ed. Chez Mequignon-Marvis, 1817.

Valladares Neto, J.; Rino Neto, J. \& Batista de Paiva, J. Orthodontic movement of teeth with short root anomaly: should it be avoided, faced or ignored? Dental Press J. Orthod., 18(6):72-85, 2013.

Valmaseda-Castellón, E.; Berini-Aytés, L. \& Gay-Escoda, C. Supernumerary premolars. Report of 10 cases. Bull. Group Int. Rech. Sci. Stomatol. Odontol., 43(1):19-25, 2001.

\author{
Dirección para Correspondencia \\ Prof. Dr. Ramón Fuentes Fernández \\ Centro de Investigación en Ciencias Odontológicas \\ Facultad de Odontología, Universidad de La Frontera \\ Av. Francisco Salazar 01145 \\ Temuco \\ CHILE
}

Email: ramon.fuentes@ufrontera.cl

Recibido : 10-06-2020

Aceptado: 09-09-2020 